\title{
NO HEAVY-ELEMENT DISPERSION IN THE GLOBULAR CLUSTER M92*
}

\author{
JUdiTH G. COHEN \\ Palomar Observatory, Mail Stop 249-17, California Institute of Technology, Pasadena, CA 91125, USA; jlc@ astro.caltech.edu \\ Received 2011 July 9; accepted 2011 September 12; published 2011 September 27
}

\begin{abstract}
Although there have been recent claims that there is a large dispersion in the abundances of the heavy neutron capture elements in the old Galactic globular cluster M92, we show that the measured dispersion for the absolute abundances of four of the rare earth elements within a sample of 12 luminous red giants in M92 ( $\leqslant 0.07$ dex) does not exceed the relevant sources of uncertainty. As expected from previous studies, the heavy elements show the signature of the $r$-process. Their abundance ratios are essentially identical to those of M30, another nearby globular cluster of similar metallicity.
\end{abstract}

Key words: Galaxy: halo - globular clusters: individual (M92, NGC 7099)

\section{INTRODUCTION}

Globular clusters are canonically viewed as stellar systems within which all stars formed with the same initial chemical inventory at approximately the same time in the distant past. More than 30 years ago, it was realized that the light elements $(\mathrm{C}, \mathrm{N}, \mathrm{O}, \mathrm{Na}, \mathrm{Mg}$, and $\mathrm{Al}$ ) show correlated star-to-star variations (see, e.g., the review by Gratton et al. 2004 and references therein). There are a few massive globular clusters that show a range of abundances among both the light and heavy elements that are believed to be remnants of accreted satellites, including $\omega$ Cen, whose anomalies were discovered more than 40 years ago (see Norris et al. 1996 and references therein), and more recently M22 (Da Costa et al. 2009; Marino et al. 2009). M15 was discovered by Sneden et al. $(1997,2000)$ to show a range of abundances among the heavy neutron capture elements, confirmed by Otsuki et al. (2006). In M15, a massive lowmetallicity Galactic globular cluster, one sees the $r$-process ratios among the heavy neutron capture elements, with a wide range of $r$-element abundances while the Fe-peak elements have constant abundances. The decoupling of the heavy neutron capture elements from the Fe peak is common among extremely metal-poor halo field stars, as was first shown by McWilliam et al. (1995); see, e.g., Figure 17 of Cohen \& Huang (2010) for a more recent view; it commences at a metallicity comparable to that of M15. Yong \& Grundahl (2008) found variations of the $s$-process elements $\mathrm{Zr}$ and La in NGC 1851, which presumably have a different origin and are more easily explained in conventional globular cluster models.

M92, a Galactic globular cluster with comparable metallicity, distance, and age as M15, is slightly less luminous. Until very recently, there was no hint in the literature of any star-to-star variations for any element heavier than $\mathrm{Al}$ in the many published spectroscopic studies of red giant stars in M92 beginning with Cohen (1979). Sneden et al. (2000) carried out the most recent study which included a substantial number of members of M92.

Very recently, Roederer \& Sneden $(2011 ; \mathrm{RS} 11)$ claim to have detected a large range $(\sim 0.6$ dex for Eu) in the heavy neutron capture elements in M92 with a sample of 19 stars observed with the Hydra spectrograph at the WIYN $3.5 \mathrm{~m}$ telescope. Their choice of this instrument requires balancing the advantage of

\footnotetext{
* Based in part on observations obtained at the W.M. Keck Observatory, which is operated jointly by the California Institute of Technology, the University of California, and the National Aeronautics and Space Administration.
}

substantial multiplexing of objects against a restricted spectral range and a moderate spectral resolution.

In the present Letter, we present evidence that there is no detectable range in the heavy neutron capture element abundances for a sample of 12 giants in M92 with considerably better spectra than those used by RS11. Our measured dispersion in abundance for each of $\mathrm{Y}, \mathrm{Ba}, \mathrm{La}$, and $\mathrm{Eu}$, as well as for $\mathrm{Fe}$, is $\leqslant 0.07 \mathrm{dex}$, which as we will show is comparable to that expected from known sources of uncertainty. This is smaller by a factor of at least four than the range claimed by RS11.

\section{STELLAR SAMPLE, OBSERVATIONS, AND ABUNDANCE ANALYSES}

To evaluate the validity of the claim of star-to-star variation in the neutron capture element abundances, we examined the spectra of luminous M92 giants with high signal-to-noise ratio $(\mathrm{S} / \mathrm{N})$ that were taken by us after the High Resolution Echelle Spectrometer (HIRES; Vogt et al. 1994) detector upgrade in early 2004. We had seven such spectra; five from 2006 April and two from 2008 June. Recently, we observed six luminous RGB stars in M92 selected from RS11 to span a large range in their claimed $[\mathrm{Eu} / \mathrm{Fe}]$. This group of stars includes one previously observed in 2006 April, for a total sample of 12 luminous RGB stars in M92. All are members of M92 based on their radial velocities and the appearance of their spectra. Details of our sample are given in Table 1.

The spectra of these M92 stars are all of high S/N with spectral resolution of 35,000 . The 2006 April spectra were taken with HIRES-B; they do not extend redder than $5950 \AA$. Since we took them to get good coverage in the UV, their $\mathrm{S} / \mathrm{N}$ at the wavelengths of interest here is very high, exceeding 150 per spectral resolution element at all relevant wavelengths. The other M92 spectra were taken with HIRES-R. They cover 3900 to $8350 \AA$. The 2011 spectra also have very high S/N except for M92 IV-10, which has a somewhat lower $\mathrm{S} / \mathrm{N}$. The 2008 April spectra of the two faintest stars have somewhat lower $\mathrm{S} / \mathrm{N}$, perhaps 80 per spectral resolution element. There are small gaps between the three CCDs that are mosaiced together to form the HIRES detector. One key line of La II, at $4086 \AA$, falls into one of those gaps for the HIRES-B spectra only.

To illustrate the high $\mathrm{S} / \mathrm{N}$ of these spectra, sections around two key rare earth features are shown in Figure 1 for three of the sample red giants. These three stars are in common with 

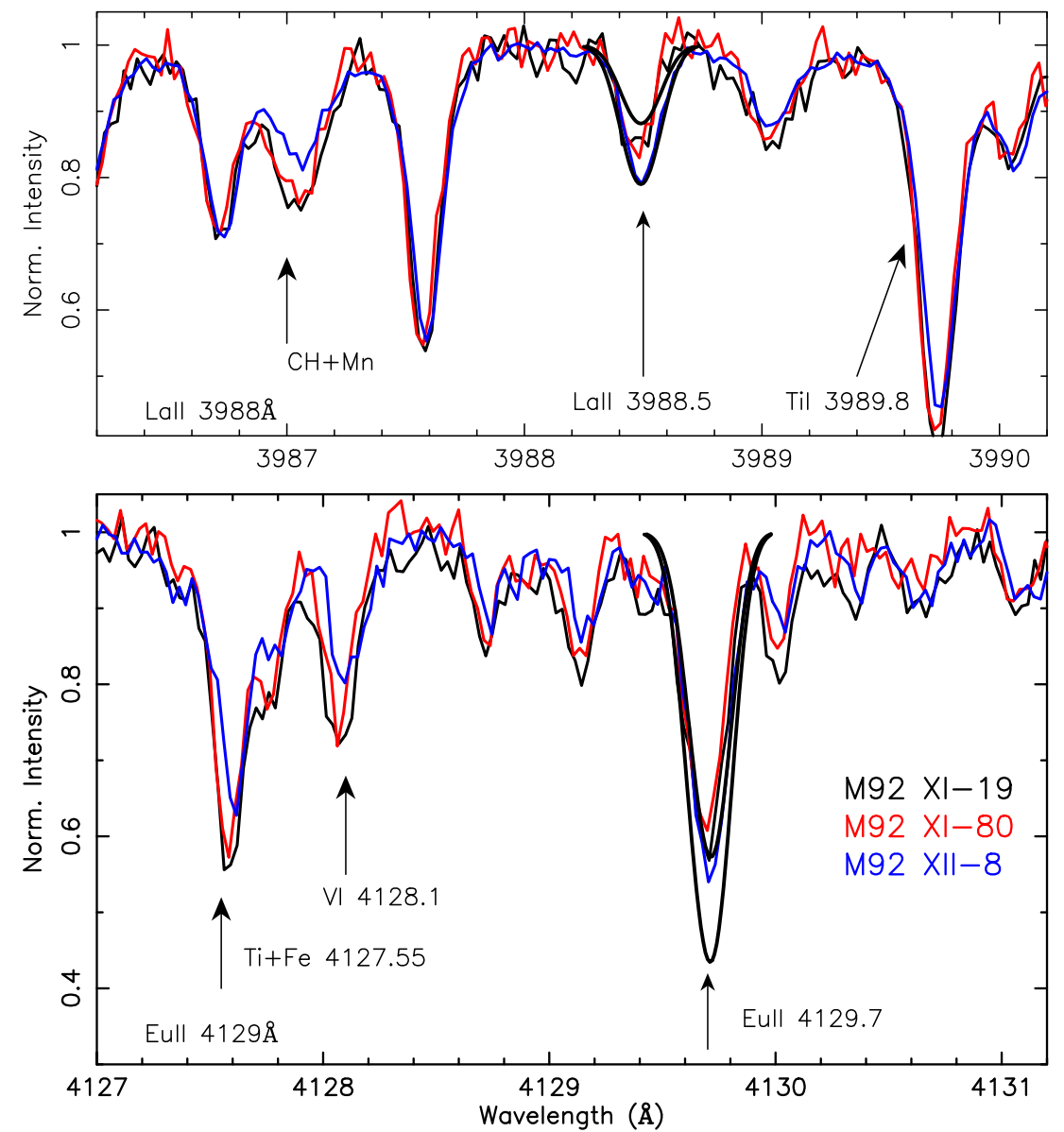

Figure 1. Spectra for the three luminous red giants M92 XI-19, XI-80, and XII- 8 are superposed for the region of the $3988 \AA$ La II line and the $4129 \AA$ Eu II line. These stars have $V$ between 12.78 and 13.09 mag. These three stars were included in the sample of RS11; spectra of two of them in the vicinity of the same La II line and a Eu II line at $3907 \AA$ are shown in their Figure 7; they claim to have detected a very large difference in rare earth abundances between the two stars. The two thick black line profiles in each panel were synthesized using abundances of La or Eu differing by 0.3 dex.

Table 1

M92 Sample for Rare Earth Study

\begin{tabular}{lccccc}
\hline \hline ID & $\begin{array}{c}V^{\mathrm{a}} \\
(\mathrm{mag})\end{array}$ & $\begin{array}{c}T_{\text {eff }}{ }^{\mathrm{b}} \\
(\mathrm{K})\end{array}$ & $\begin{array}{c}\log (g) \\
(\mathrm{dex})\end{array}$ & $\begin{array}{c}v_{t} \\
\left(\mathrm{~km} \mathrm{~s}^{-1}\right)\end{array}$ & {$[\mathrm{Fe} 12 / \mathrm{H}]^{\mathrm{c}}$} \\
\hline X-49 & 12.31 & $4320(25)$ & 0.68 & 2.1 & 5.22 \\
III-65 & 12.42 & $4454(30)$ & 0.83 & 2.3 & 5.14 \\
II-53 & 12.45 & $4458(33)$ & 0.84 & 2.3 & 5.16 \\
XII-8 & 12.78 & $4520(25)$ & 1.02 & 2.1 & 5.04 \\
XI-80 & $12.82^{\mathrm{d}}$ & $4532(35)$ & 1.12 & 2.0 & 5.17 \\
XI-19 & $13.09^{\mathrm{d}}$ & $4560(35)$ & 1.07 & 2.0 & 5.23 \\
IV-10 & $13.46^{\mathrm{d}}$ & $4614(33)$ & 1.35 & 2.0 & 5.20 \\
III-82 & 13.30 & $4630(30)$ & 1.31 & 2.0 & 5.10 \\
IV-79 & 13.45 & $4689(37)$ & 1.39 & 1.9 & 5.12 \\
XII-34 & 14.33 & $4722(58)$ & 1.40 & 2.0 & 5.14 \\
X-20 & 15.57 & $5183(35)$ & 2.48 & 1.5 & 5.16 \\
VI-90 & 15.69 & $5215(24)$ & 2.53 & 1.6 & 5.13 \\
\hline
\end{tabular}

Notes.

${ }^{\text {a }}$ Photometry from Stetson (2005).

b The dispersion around the mean $T_{\text {eff }}$ from the three colors used is given in parentheses.

${ }^{c}$ The average of $[\mathrm{Fe} / \mathrm{H}]$ from neutral and from ionized $\mathrm{Fe}$ lines.

d Photometry from Buonanno et al. (1983).

the sample of RS11, who claim that they show a large range of rare earth abundances. The two thick black curves are spectral syntheses for the two features with the abundance of the relevant element, La or Eu, altered by 0.3 dex.
We selected the strongest and least crowded lines of Y II, Ba II, La II, and Eu II within the wavelength regime covered. We chose the $5854 \AA$ line of Ba II instead of the stronger line at $4554 \AA$ as it is very strong in the coolest stars. Hyperfine structure (HFS) patterns for Ba II are from McWilliam (1998). For Eu II, we chose the resonance line at $4129 \AA$ as it is both stronger and less crowded than the bluer Eu II lines including that used by RS11 at $3907 \AA$. The transition probability and HFS are from Lawler et al. (2001b). The Eu II lines are partially resolved due to the wide HFS pattern; the HFS correction for the $4129 \AA$ line ranges from -1.1 dex for the coolest M92 giant in our sample to zero for the weak line seen in the hottest giants in our sample. The wide HFS pattern for Eu explains why the change in line strength shown in Figure 1 for a line well above the linear part of the curve of growth is so large for a 0.3 dex change in $\mathrm{Eu}$ abundance. We chose La II $3988 \AA$, one of the four lines used by RS11, with a $g f$ value and HFS pattern from Lawler et al. (2001a). This line is too weak to measure reliably in the two hottest stars in our sample as well as in M92 IV-10. Among the cooler M92 giants, the HFS correction for the $3988 \AA$ A feature of La II varies from -0.17 dex to -0.07 dex. The $g f$ values for the two Y II lines are taken from the current version of the National Institute of Standards and Technology (Ralchenko et al. 2010). HFS corrections are not needed for Y II (Hannaford et al. 1983).

We measured equivalent widths for the same small set of rare earth lines in each of the M92 giants. The agreement of the measured $W_{\lambda}$ from the two spectra of M92 XII-8 taken 
Table 2

Statistics for Absolute Abundances of Rare Earths in M92

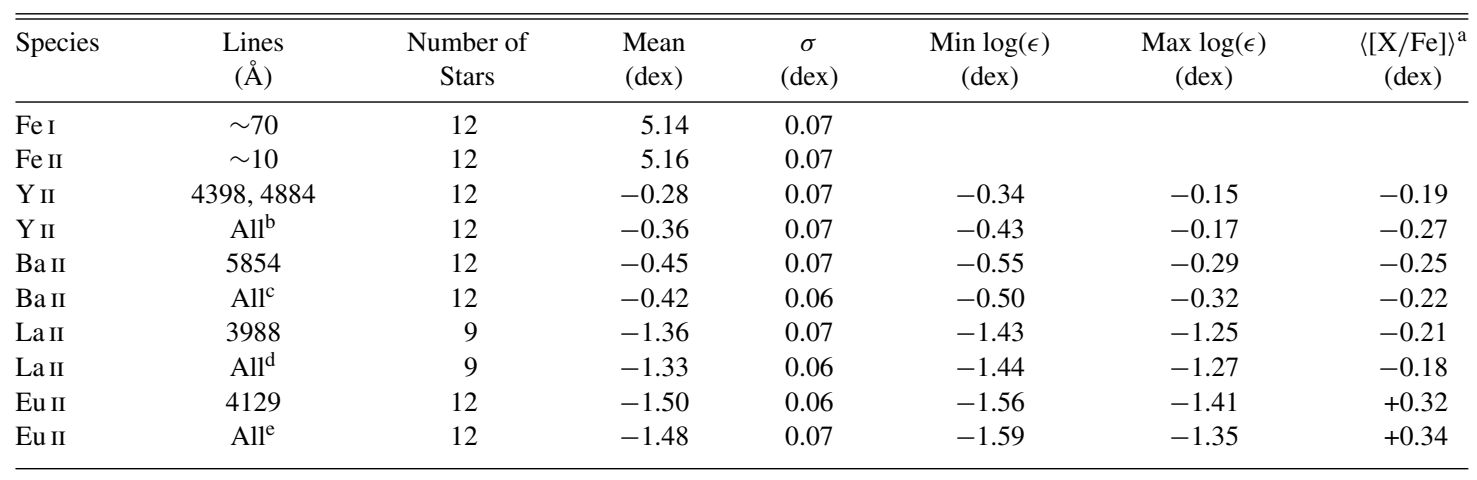

Notes.

a Assumes $[\mathrm{Fe} / \mathrm{H}](\mathrm{M} 92)=-2.33$ dex.

${ }^{\mathrm{b}} \mathrm{Up}$ to eight $\mathrm{Y}$ II lines.

c The two bluer or all four of 4554, 5854, 6141, and $6196 \AA$ lines.

${ }^{\mathrm{d}}$ Up to seven La II lines.

e All detected among the three Eu II lines 3905, 4129, and $6645 \AA$.

five years apart is such that we adopt an uncertainty in these measurements of $3 \mathrm{m \AA}$. For the two warmer stars which are more than 2 mag fainter in $V$ and have much weaker rare earth lines we adopt $4 \mathrm{~m} \AA$ as the uncertainty in $W_{\lambda}$. The region of the $4129 \AA$ line of Eu II becomes rather crowded in the two coolest stars and its $W_{\lambda}$ is somewhat more uncertain for them. We also measured equivalent widths of any other lines of these four elements that we could detect. HFS patterns from the sources given above were used for all lines of Ba II, La II, and Eu II; $r$-process isotopic ratios were assumed.

The abundance analysis follows our previously published work; see, e.g., Cohen \& Melendez (2005). We use a photometric definition for $T_{\text {eff }}$ using $V-I, V-J$, and $V-K_{s}$, where the optical colors are from Stetson (2005) and the infrared ones from the Two Micron All Sky Survey (Skrutskie et al. 2006; Cutri et al. 2003). We use the predicted color grid of Houdashelt et al. (2000). We adopt the mean of the three resulting values of $T_{\text {eff }}$ for our abundance analysis, then derive a surface gravity assuming a mass along the RGB of $0.8 M_{\odot}$, with the distance and interstellar absorption to M92 taken from Harris (1996). We adopt these computed values without modification for all stars. The resulting stellar parameters, and the dispersions of the three values for $T_{\text {eff }}$, are given in Table 1. We use 1D LTE stellar model atmospheres from Kurucz (1993) with the abundance analysis code MOOG (Sneden 1973). The microturbulent velocity $v_{t}$ is set by requiring the derived abundance for individual $\mathrm{Fe}$ I lines to be independent of the equivalent width.

This is a differential analysis from star to star within M92. The uncertainties for the absolute abundances of the rare earths for each star will have a term representing the uncertainty of the relative $T_{\text {eff }}$, a contribution from the uncertainty in the equivalent widths, and a contribution from a possible small error in $v_{t}$. We assign an uncertainty of $30 \mathrm{~K}$ to the $T_{\text {eff }}$ based on the dispersion in this parameter as determined from the various available colors given in Table 1 . This translates into an uncertainty in the absolute abundances for the rare earth elements under consideration of a maximum of $0.04 \mathrm{dex}$, including the contribution from the matching $\log (g)$ change. The contribution for an error in $v_{t}$ of $0.1 \mathrm{~km} \mathrm{~s}^{-1}$ depends on the line strength and whether or not HFS corrections apply. It appears to be largest for $5854 \AA$ Ba II line (0.07 dex) and drops to $0.02 \mathrm{dex}$ for the other rare earth species under consideration.
For the two warmer stars, the dominant uncertainty arises from the $4 \mathrm{~m} \AA$ allowance for errors in the measured equivalent widths. Summing the three terms in quadrature, for these two stars, we adopt an uncertainty in absolute abundance for the rare earth species studied here of 0.13 dex. For the cooler stars, the three terms contribute roughly equally, and we adopt an uncertainty in absolute abundances of 0.08 dex for the species of interest. We increase the overall uncertainty in absolute abundances by $15 \%$ for the three M92 giants not included in the photometric database of Stetson (2005).

Table 2 provides statistics for the absolute abundances of these four rare earths in our sample of M92 luminous giants both for only the best lines and for when all available lines are used. The maximum dispersion is only 0.07 dex for these four elements within the 12 stars studied in either case. The dispersion for the Fe abundance as derived from either the neutral or singly ionized species is also $0.07 \mathrm{dex}$.

The derived absolute abundances of $\mathrm{Y}, \mathrm{Ba}, \mathrm{La}$, and $\mathrm{Eu}$ for each of the 12 luminous red giants in M92 using the best lines for each of these elements are shown in Figure 2. Figure 3 shows the derived absolute abundance of Eu versus that of Ba. There is no sign of any correlation. Furthermore, a comparison of the synthesized and observed line profiles for three stars with very close $T_{\text {eff }}$ for the $4129 \AA$ Eu II and the $3988 \AA$ La II lines (Figure 1) again suggests very strongly that the range of abundance in the heavy neutron capture elements among the M92 sample giants must be small.

On the basis of this evidence, we conclude that the abundances of each of these four heavy neutron capture elements are constant from star to star within our sample to within the expected uncertainties discussed above. The almost constant Eu abundance derived from the $4129 \AA$ line is very gratifying given the very large HFS correction for this strong line in the coolest sample stars, while it is negligible in the faintest (hence warmest) two stars. The same comment applies for the four Ba II lines used, as the line at $4554 \AA$ has a substantial HFS correction while the others do not.

It is interesting to compare the abundance ratios for these four heavy neutron capture elements with those of other Galactic globular clusters of similar metallicity. J. Cohen and collaborators (Cohen et al. 2011) have recently carried out in an identical manner a detailed abundance analysis for a sample of 


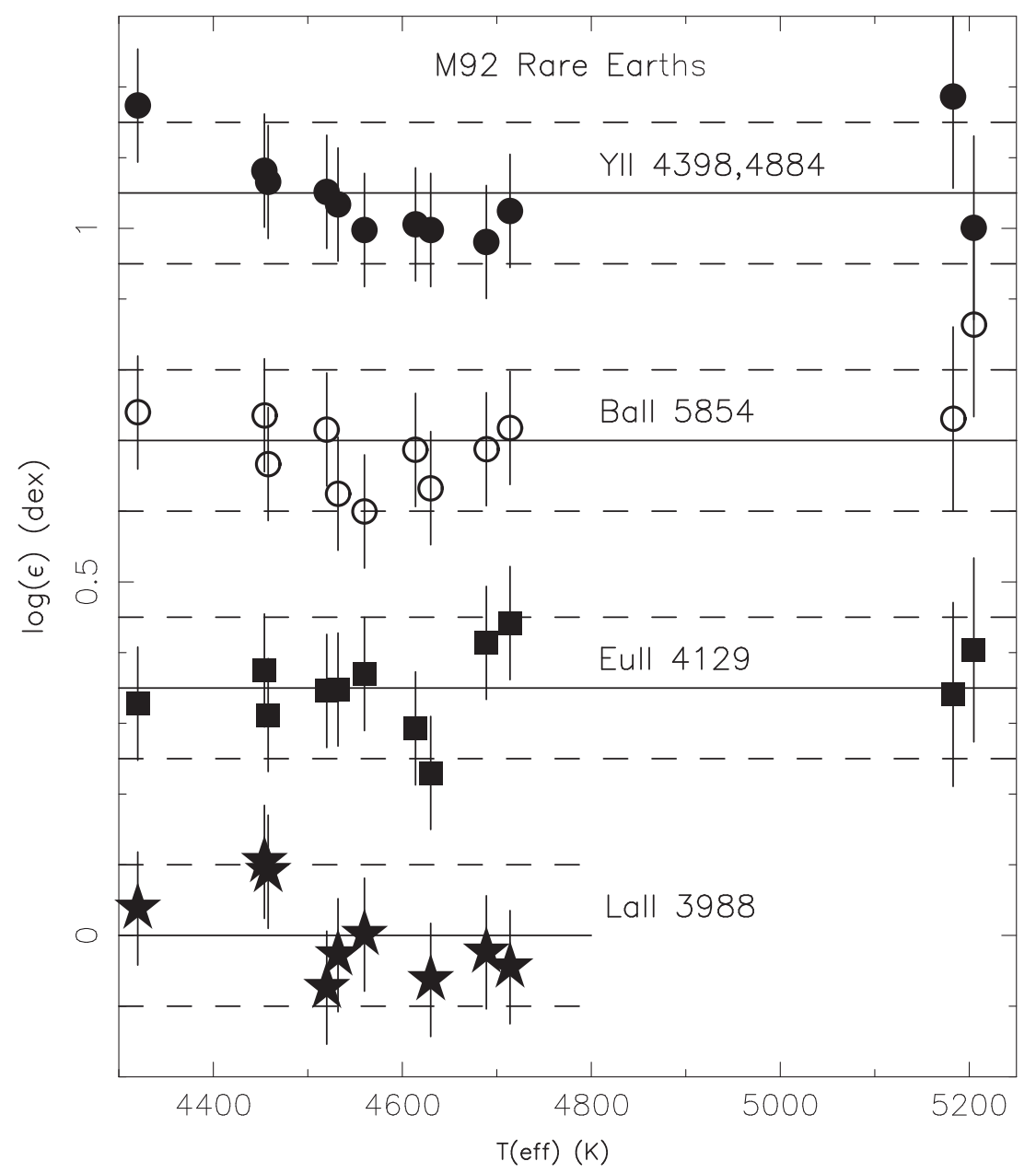

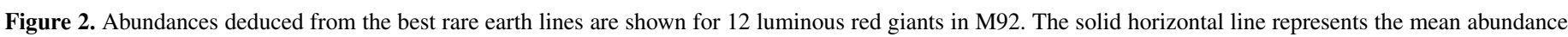
of the element, while the dashed lines are offset above and below the mean by 0.1 dex. The vertical axis values are offset by a constant between each line.

luminous red giants from another nearby metal-poor old Galactic GC, M30. Their $[\mathrm{Fe} / \mathrm{H}]$ values are almost the same, -2.33 dex for M92 versus -2.40 dex for M30. Their ratios $\langle[\mathrm{Y} / \mathrm{Fe}]\rangle$ and $\langle[\mathrm{Ba} / \mathrm{Fe}]\rangle$ are identical to within 0.04 dex. That for $[\mathrm{La} / \mathrm{Fe}]$ is more discrepant, but the La II lines are weak in such metal-poor stars, and the resulting La abundances are uncertain unless the spectra are of very high $\mathrm{S} / \mathrm{N}$. $\langle[\mathrm{Eu} / \mathrm{Fe}]\rangle$ differs only by 0.09 dex. For each of these four heavy neutron capture elements, the ratios agree to within the uncertainties between the M92 sample presented here and the M30 sample of Cohen et al. (2011).

The neutron capture element abundances in both M92 and in M30 are indicative of the $r$-process. The high enhancement of $[\mathrm{Eu} / \mathrm{Fe}]$, about +0.5 dex above that of $[\mathrm{Y}, \mathrm{Ba}, \mathrm{La} / \mathrm{Fe}]$, signals that the $r$-process dominates in M92, as is the case in other very metal-poor globular clusters (see, e.g., Gratton et al. 2004).

\section{COMPARISON WITH THE WORK OF ROEDERER \& SNEDEN}

The sample of RS11 is somewhat larger than ours, 19 versus 12 M92 red giants. However, only 16 of the 19 stars in their sample actually have measured Eu abundances; all 19 have Y and La abundances. There are no suitable $\mathrm{Ba}$ lines within the limited spectral coverage of the RS11 spectra. Six of the twelve M92 red giants in our sample are in common with that of RS11. One of these, M92 IV-10, has a very low S/N spectrum in RS11; no abundances are tabulated in their paper for this star. For the other five stars in common, we find a total range in $\log [\epsilon(\mathrm{Eu})]$ of 0.11 dex, while they find a total range of 0.44 dex. For La II, our range for these five stars is only $0.07 \mathrm{dex}$, while their total range is 0.19 dex. RS11 state that the $\mathrm{Y}$ abundance, inferred from two Y II lines, within their M92 sample is homogeneous; our result is in agreement with theirs.

We thus have a major disagreement on the range of the Eu and, to a lesser extent, of the La abundances within the respective samples of M92 giants between us and the very recent work of RS11. We examine the data and the analysis techniques used by these two groups in an attempt to isolate the cause of this disagreement. The major difference between the present work and that of RS11 is the higher quality of our Keck spectra. Their wavelength range was restricted to $3850-4050 \AA$ with a spectral resolution of 14,000. Spectra with high $\mathrm{S} / \mathrm{N}$ and high spectral resolution are key in any effort to work on these elements with only a few detectable lines, most of which are rather weak.

Our techniques for stellar parameter determination are similar to those of RS11. However, we rely on the highly accurate photometric database of Stetson (2005) to determine $T_{\text {eff }}$ whereas they rely on the earlier photographic study by Buonanno et al. (1983). Three stars in our sample chosen for observation in the early summer of 2011 to increase overlap with the sample of RS11 are not in the Stetson database. For these we resorted to the photometry of Buonanno et al. (1983). A check of a sample of stars in common between these two photometric studies shows 


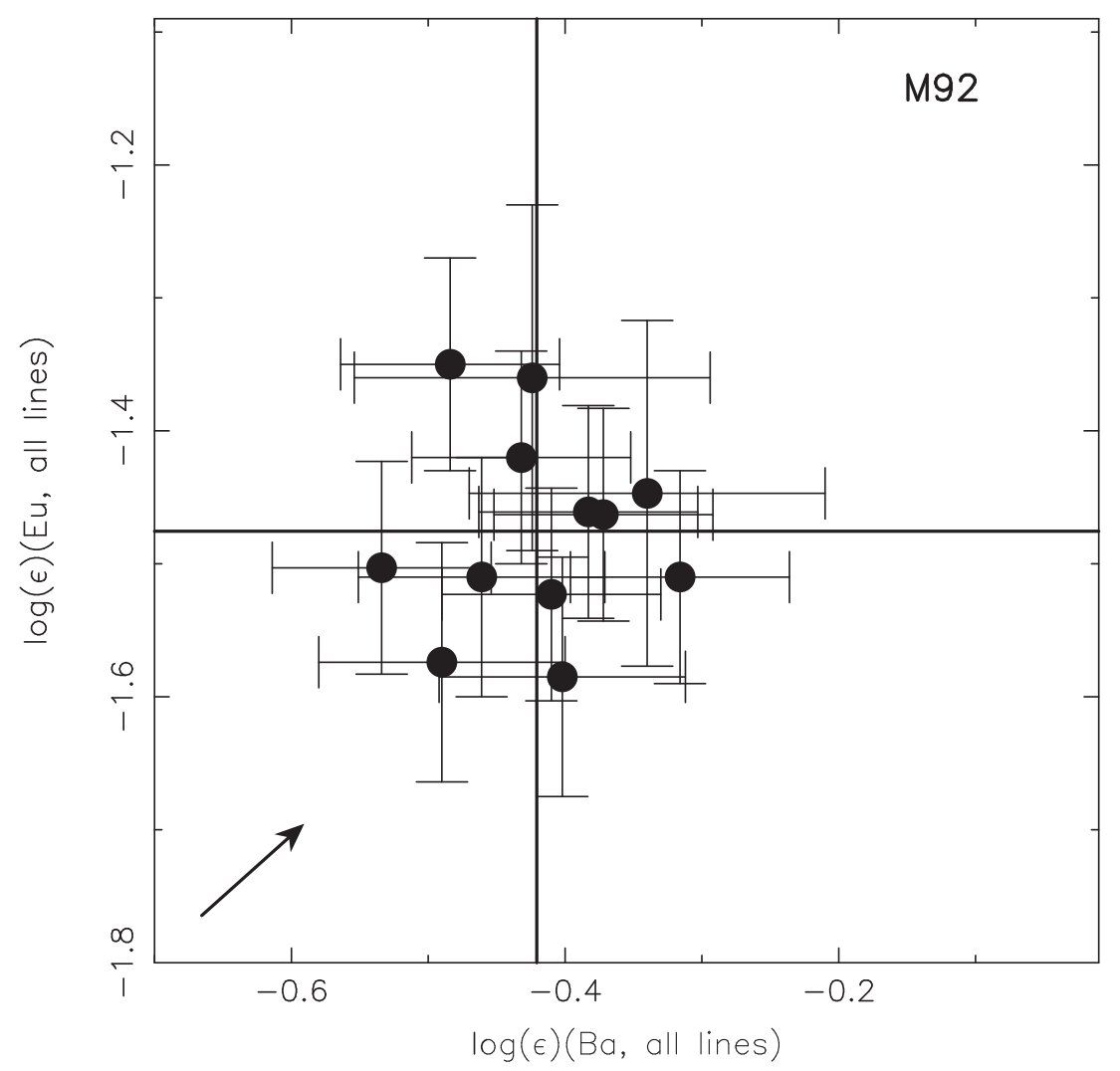

Figure 3. Ba vs. Eu abundances are shown for our M92 sample. The arrow at the lower left indicates the effect of a $T_{\text {eff }}$ increase of $50 \mathrm{~K}$.

that the old photographic photometry of Buonanno et al. (1983) is systematically fainter in $V$ by $\sim 0.06 \mathrm{mag}$, with a dispersion of 0.03 mag. The mean difference in $V$ corresponds to a $T_{\text {eff }}$ change of $50 \mathrm{~K}$ for $V-K(75 \mathrm{~K}$ for $V-J)$. Use of the older photometry will thus yield slightly lower $T_{\text {eff }}$ values for a given star. Furthermore for these three stars, identified in Table 1, there is no $I$ photometry, suggesting the possibility of larger uncertainties in $T_{\text {eff }}$ for them. The older photometry includes $B$, but we ignore $B-V$ colors as they lack sufficient sensitivity to $T_{\text {eff }}$ and are not suitable for our purpose.

A detailed star-by-star comparison of the stellar parameters between the present work and that of RS11 suggests that there is a systematic difference in the $T_{\text {eff }}$ scale with their being somewhat cooler by $\sim 60 \mathrm{~K}$. However, in both cases, the goal is a differential analysis from star to star within M92, and thus this small systematic difference is irrelevant for present purposes. We have also checked our HFS patterns versus those of RS11 and found that they are essentially identical.

We must thus conclude that the higher quality of the Keck HIRES spectra has enabled us to reach a level of accuracy which was not possible with the lower dispersion and limited spectral range of the RS11 sample. They claim detection of a "clear star-to-star dispersion spanning 0.5-0.8 dex" in La, Eu, and Ho within their M92 sample. We have demonstrated that this is not correct for either $\mathrm{La}$ or $\mathrm{Eu}$. Ho has even weaker and more blended lines, so their claimed high dispersion of Ho within M92 must be regarded with suspicion.

\section{DISCUSSION}

We have established, in contradiction to the results of RS11, that there is no detectable range in star-to-star abundance of the heavy neutron capture elements Y, Ba, La, and Eu in M92 within our sample of 12 red giant cluster members, which spans a range of $3.4 \mathrm{mag}$ in $V$ and $\sim 900 \mathrm{~K}$ in $T_{\text {eff }}$. Our dispersions in absolute abundances for these four elements are $\leqslant 0.07$ dex, consistent with the expected observational and analysis uncertainties. This is very gratifying given that some of the key spectral features used have very wide HFS patterns and hence HFS corrections which strongly vary within our sample of M92 giants. These elements show an $r$-process abundance distribution, as expected. The heavy neutron capture elements within M92 show a chemical inventory very similar to that of M30, a slightly more metal-poor and slightly less massive Galactic globular cluster.

So the remaining question is why is M15 the only globular cluster known to date within which the $r$-process dominates and a dispersion of heavy neutron capture element abundances exists. Beyond invoking the high luminosity and mass of M15, there is as yet no obvious answer. A search for abundance dispersions within massive but less well-studied globular clusters is now underway.

We are grateful to the many people who have worked to make the Keck Telescope and its instruments a reality and to operate and maintain the Keck Observatory. The author wishes to extend special thanks to those of Hawaiian ancestry on whose sacred mountain we are privileged to be guests. Without their generous hospitality, none of the observations presented herein would have been possible. The author thanks NSF Grant AST-0908139 for partial support.

\section{REFERENCES}

Buonanno, R., Buscema, G., Corsi, C. E., et al. 1983, A\&AS, 53, 1 Cohen, J. G. 1979, ApJ, 231, 751 
Cohen, J. G., \& Huang, W. 2010, ApJ, 719, 931

Cohen, J. G., Huang, W., \& Kirby, E. N. 2011, ApJ, in press

Cohen, J. G., \& Melendez, J. 2005, AJ, 129, 303

Cutri, R. M., Skrutskie, M. F., Van Dyk, S., et al. 2003, Explanatory Supplement to the 2MASS All-Sky Data Release, http://www.ipac.caltech.edu/2mass/ releases/allsky/doc/explsup.html

Da Costa, G. S., Held, E. V., Saviane, I., \& Gullieuszik, M. 2010, ApJ, 705 1481

Gratton, R., Sneden, C., \& Carretta, E. 2004, ARA\&A, 42, 385

Hannaford, P., Lowe, R. M., Grevesse, N., Biemont, E., \& Whaling, W. 1982, ApJ, 261, 736

Harris, W. E. 1996, AJ, 112, 1487

Houdashelt, M. L., Bell, R. A., \& Sweigart, A. V. 2000, AJ, 119, 1448

Kurucz, R. L. 1993, Kurucz CD-ROM 13, ATLAS 9 Stellar Atmosphere Programs and $2 \mathrm{~km} / \mathrm{s}$ Grid (Cambridge: SAO)

Lawler, J. E., Bonvallet, G., \& Sneden, C. 2001a, ApJ, 556, 452

Lawler, J. E., Wickliffe, M. E., Den Hartog, E. A., \& Sneden, C. 2001b, ApJ, 563,1075
Marino, A. F., Milone, A. P., Piotto, G., et al. 2009, A\&A, 505, 1099

McWilliam, A. 1998, AJ, 115, 1640

McWilliam, A., Preston, G. W., Sneden, C., \& Searle, L. 1995, AJ, 109, 2757

Norris, J. E., Freeman, K. C., \& Mighell, K. J. 1996, ApJ, 462, 241

Otsuki, K., Honda, S., Aoki, W., Kajino, T., \& Matthews, G. J. 2006, ApJ, 641, L117

Ralchencko, Yu., Kramida, A. E., Reader, J., \& The NIST ASD Team 2010, NIST Atomic Spectra Database, version 4.0, http://physics.nist.gov/asd

Roederer, I. U., \& Sneden, C. 2011, AJ, 142, 22 (RS11)

Skrutskie, M. F., Cutri, R. M., Stiening, R., et al. 2006, AJ, 131, 1163

Sneden, C. 1973, PhD thesis, Univ. Texas

Sneden, C., Kraft, R. P., Shetrone, M. D., et al. 1997, AJ, 114, 1964

Sneden, C., Pilachowski, C. A., \& Kraft, R. P. 2000, AJ, 120, 1351

Stetson, P. B. 2005, PASP, 117, 563

Vogt, S. E., Allen, S. L., Bigelow, B. C., et al. 1994, Proc. SPIE, 2198, 362

Yong, D., \& Grundahl, F. 2008, ApJ, 672, 29 